\title{
EDITORIAL AND COMMENT \\ Evaluating the Risks of Opioid Use for Chronic Pain: Moving Beyond Overdose
}

\author{
Stephen G. Henry, MD \\ Department of Internal Medicine, University of California Davis School of Medicine, Sacramento, CA, USA.
}

KEY WORDS: osteoarthritis; opioid analgesics; falls; fractures; men. $\mathrm{J}$ Gen Intern Med 31(5):453-4

DOI: $10.1007 / \mathrm{s} 11606-016-3659-5$

(C) Society of General Internal Medicine 2016

$\mathrm{T}$ he dramatic increase in use of prescription opioids to treat chronic pain and the subsequent rise in opioid-related overdose and death have become a major focus of public health efforts at the state and national levels. Between 2001 and 2014, the rate of deaths related to prescription opioids rose from 1.9 to 5.9 per $100,000 .{ }^{1}$ In response, the CDC has taken the unusual step of proposing clinical guidelines for prescribing opioids to treat chronic pain. On February 2, 2016, President Obama proposed additional funding of 1.1 billion dollars to address prescription opioid and heroin abuse.

The problems of prescription opioid overdose and abuse are real and critically important to address. At the same time, the risk of overdose is concentrated among a relatively small proportion of patients who consume high doses $(>100 \mathrm{mg}$ morphine equivalents per day), or who are at high-risk for other reasons (e.g., patients with substance use disorders). The top $10 \%$ of patients taking opioids account for $>60 \%$ of all opioids consumed in the United States. ${ }^{2}$ In contrast to growing evidence of a dose-response relationship between opioid consumption and overdose risk, we know relatively little about other risks of long-term opioid use, including risks that are clinically important for the majority of patients on opioids, who are taking low doses of these medications. ${ }^{3}$ Falls and fractures are particularly important potential risks because they are major contributors to disability and death among older adults. ${ }^{4}$ Older patients with osteoarthritis pain and contraindications to nonsteroidal anti-inflammatory drugs are a clinical population who are at risk for falls and for whom low-dose opioids may sometimes be a reasonable treatment option.

The article by Krebs et al. published in this issue of JGIM is a welcome addition to the literature characterizing associations between opioid use and risk of fall or fracture. ${ }^{5}$ The authors performed a secondary analysis of data from the Osteoporotic Fractures in Men Study (MrOS), a multisite cohort study that enrolled 5994 ambulatory, community-dwelling men aged 65 years or older between 2000 and 2002. ${ }^{6}$ Detailed clinical measures related to pain, bone density, health, and functional status were obtained from participants during one baseline and two subsequent clinic visits over a mean follow-up period of

Published online March 7, 2016
4.5 years. The primary outcomes of interest, participantreported falls and fracture events, were assessed every 4 months; fractures were confirmed radiographically. The authors analyzed a subset of 2902 patients who reported persistent back, knee, or hip pain at baseline, in order to test their hypothesis that opioid use (defined as taking opioid analgesics daily or near daily) would be associated with increased risk of falls and incident fractures.

In their primary analysis, the authors found that, compared to no opioid use, daily or near daily opioid use was not associated with a statistically significant increased risk of either fall (RR 1.10; $95 \%$ CI 0.99-1.24, $p=0.08$ ) or incident fracture (HR 1.13, $95 \%$ CI $0.94-1.36 . p=0.07$ ). In secondary analyses, they found no significant difference between these two groups in rates of either hip fracture or decline in physical performance over time. The authors concluded that additional data and research are needed to determine whether opioid use is associated with risk of falls, fractures, and functional impairment among older adults suffering from musculoskeletal pain. The parent study was restricted to men, so these findings do not provide information about the association between opioid use and risk of falls or fractures among older women.

This study has several notable strengths. The parent study followed a large number of older men for up to 9 years and had remarkably complete follow-up data. Participants provided detailed clinical information that cannot be obtained from administrative records. The primary outcomes were prospectively and rigorously measured. This study is also one of the few to examine associations between opioid use and falls, which, unlike fractures, do not reliably result in patients seeking formal medical care.

The authors' statistical analyses were rigorous and made efficient use of the parent study's repeated-measures design. They analyzed opioid use, falls, and even propensity scores as time-varying factors to account for participants whose selfreported opioid use changed during the study. Their results also show the utility of using propensity scores to adjust for differences in measured confounders when attempting to make causal inference from observational data. ${ }^{7}$ Participants who reported opioid use at baseline differed substantially from those who did not in several respects: they were less likely to report good or excellent health, were frailer, and were more likely to report back pain. Propensity score techniques attempt to account for these differences and are most useful for studies such as this one that include detailed clinical information on all 
participants. The authors also demonstrate the importance of restricting propensity score analyses to participants who have a meaningful probability of being assigned to either group (i.e., either the opioid or non-opioid group). Based on observed characteristics, one-sixth of study participants had a negligible probability of being prescribed an opioid and so were excluded from the primary propensity score analysis. The association between opioid use and falls would have been statistically significant if patients with low propensity scores were included; that association is likely to be misleading because it would have included a large number of participants who had no real probability of receiving opioid prescriptions.

These negative findings contrast with several prior studies that reported positive associations between opioid use and fracture risk. ${ }^{8,9}$ This discrepancy may relate to the lack of data on the dose or duration of opioid use in the current study. If opioid-related fracture risk is dose-dependent and (like overdose risk) is concentrated among a relatively small proportion of patients taking opioids, the lack of data on dose would substantially reduce the study's power to detect a true association. The results may also have been affected by historical changes in clinical practice. The parent study collected data from 2000 to 2009, a period which saw substantial increases in opioid prescribing for chronic pain. ${ }^{2}$ The observed increase in prevalent opioid use from $4.7 \%$ at baseline to $10.5 \%$ at the final clinic visit is consistent with this possibility.

On the other hand, the findings reported by Krebs et al. are less likely to be influenced by a small group of high-risk participants, and so may be more applicable to the median older man taking low-dose opioids for musculoskeletal pain.

This study highlights two important challenges facing researchers examining the risks and benefits of opioids for chronic pain. First, as the baseline data from the current study make clear, patients who take opioids for chronic pain are different from patients who do not in many important ways that can be difficult to measure. These differences include not only the health status measures reported in the current study, but also patients' clinical response to opioids, as well as psychological and mental health factors, including depression and past history of substance abuse. Prior cohort studies have identified a phenomenon known as "adverse selection," whereby patients at the greatest risk of opioid-related harms are most likely to receive long-term opioids. ${ }^{10}$

Second, while the much-needed focus on opioid-related overdose has greatly expanded clinicians' ability to identify patients at risk of being harmed by opioids, there is relatively less data on which patients, if any, are reasonably likely to benefit from the use of low-dose opioids for musculoskeletal pain. Among patients at low risk for overdose and abuse, both the risks and benefits of opioids are likely to be modest.
Therefore, to identify patients who are reasonable candidates for opioid therapy, clinicians need better data on benefits as well as on risks other than overdose and death (e.g., falls, fractures, endocrine dysfunction, and depression) in this population. Given the limitations of observational data and the phenomenon of adverse selection, clinical trials testing lowdose opioid use in carefully selected patients will likely be required to provide this information. Such trials are unlikely to be a priority while opioid overdose and death remain such high-profile problems. In the meantime, the study by Krebs et al. provides much-needed additional information about the risks and benefits of using opioids to treat chronic musculoskeletal pain.

Corresponding Author: Stephen G. Henry, MD; Department of Internal Medicine University of California Davis School of Medicine, 4150 V Street, Suite 2400, Sacramento, CA 95817, USA (e-mail: sghenry@ucdavis.edu).

\section{Compliance with Ethical Standards:}

Conflict of Interest: Dr. Henry is supported by a KL2 award from NCATS (UL1 TROOOOO2 / KL2 TROOO134) and previously received support from an educational grant from the ER/LA Opioid Analgesics REMS Program Companies.

\section{REFERENCES}

1. Rudd R, Aleshire N, Zibbell JE, Gladden RM. Increases in drug and opioid overdose deaths-United States, 2000-2014. MMWR. 2016;64(50/51):1378-1382.

2. Edlund MJ, Martin BC, Fan MY, Braden JB, Devries A, Sullivan MD. An analysis of heavy utilizers of opioids for chronic noncancer pain in the TROUP study. J Pain Symptom Manag. 2010;40(2):279-289.

3. Chou R, Turner JA, Devine EB, et al. The effectiveness and risks of longterm opioid therapy for chronic pain: a systematic review for a national institutes of health pathways to prevention workshop. Ann Intern Med. 2015;162(4):276-286.

4. Kannus P, Sievanen H, Palvanen M, Jarvinen T, Parkkari J. Prevention of falls and consequent injuries in elderly people. Lancet. 2005;366(9500): 1885-1893.

5. Krebs EE, Paudel M, Taylor BC, et al. Association of opioids with falls, fractures, and physical performance among older men with persistent musculoskeletal pain. J Gen Intern Med. doi:10.1007/s11606-015-3579-9

6. Orwoll E, Blank JB, Barrett-Connor E, et al. Design and baseline characteristics of the osteoporotic fractures in men (MrOS) study-a large observational study of the determinants of fracture in older men. Contemp Clin Trials. 2005;26(5):569-585.

7. Rubin DB. Estimating causal effects from large data sets using propensity scores. Ann Intern Med. 1997;127(8 Pt 2):757-763.

8. Saunders KW, Dunn KM, Merrill JO, et al. Relationship of opioid use and dosage levels to fractures in older chronic pain patients. J Gen Intern Med. 2010;25(4):310-315.

9. Li L, Setoguchi S, Cabral H, Jick S. Opioid use for noncancer pain and risk of fracture in adults: a nested case-control study using the general practice research database. Am J Epidemiol. 2013; 178(4):559-569.

10. Sullivan MD, Ballantyne JC. What are we treating with long-term opioid therapy? Arch Intern Med. 2012;172(5):433-434. 